\title{
High Resolution Mid-Infrared Up-Conversion LIDAR
}

\author{
Max Widarsson ${ }^{1}$, Markus Henriksson ${ }^{2}$. Patrick Mutter ${ }^{1}$, Carlota Canalias $^{1}$, Valdas Pasiskevicius ${ }^{1}$, and \\ Fredrik Laurell ${ }^{1}$ \\ ${ }^{I}$ Department of Applied Physics, Royal Institute of Technology, Roslagstullsbacken 21, 10691 Stockholm, Sweden \\ ${ }_{2}^{2}$ Swedish Defence Research Agency, Olaus Magnus Väg 42, 58330 Linköping, Sweden
}

Light in the mid-infrared (MIR) has a higher transmission through certain media, such as fog and smoke [1], than visible/near-infrared due to the lower scattering probability. Therefore it is of interest to have LIDARs operating in this spectral region, specifically utilizing photon counting, which yields high sensitivity. However, most photon counting LIDAR systems operate in the near infrared [2-4]. This is due to a lack of good detectors in the MIR.

By up-converting the MIR photons to the working regime of established Si-detectors, it is possible to reap the benefits from both the MIR and good detectors. This technique has previously successfully demonstrated detection of MIR both through a single pass configuration [5] or when the nonlinear crystal was inside a laser cavity [6].

This experiment was performed to extend the wavelength of the up-conversion MIR LIDAR to $2.4 \mu \mathrm{m}$ and achieve high spatial resolution compared to previous up-conversion photon counting LIDARs [7,8]. In this work the nonlinear up-conversion was done inside a Nd: $\mathrm{YVO}_{4}$ cavity lasing at $1064 \mathrm{~nm}$ generating light at $737 \mathrm{~nm}$.

The LIDAR was performed with the time of flight technique. To do this there were three main components of the setup, the MIR pulses, the conversion cavity and the detector for the up-converted light. The MIR pulses were reflected off a microscope slide, up-converted in the conversion cavity, spectrally filtered and detected. The MIR pulses had a temporal width below $100 \mathrm{fs}$ with a spectral bandwidth of roughly $300 \mathrm{~nm}$ centered around 2.4 $\mu \mathrm{m}$ with a repetition rate of $1 \mathrm{kHz}$ and pulse energy in the $\mathrm{pJ}$ regime.

A $10 \mathrm{~mm}$ long (9 mm grating length) periodically-poled rubidium-doped $\mathrm{KTiOPO}_{4}(\mathrm{PPRKTP})$ with a 23.5 $\mu \mathrm{m}$ period was fabricated in-house and used for the up-conversion. The crystal was inserted into a straight $\mathrm{Nd}: \mathrm{YVO}_{4}$ laser cavity pumped by a $2.5 \mathrm{~W} 808 \mathrm{~nm}$ laser diode. The PPRKTP crystal was uncoated which resulted in a relatively low intra-cavity power of $5 \mathrm{~W}$ at $1064 \mathrm{~nm}$.

A conventional silicon single-photon avalanche photodiode (Si-SPAD) with a detection efficiency of $23 \%$ at $737 \mathrm{~nm}$ was used in combination with a time tagging unit with bin widths down to $4 \mathrm{ps}$.
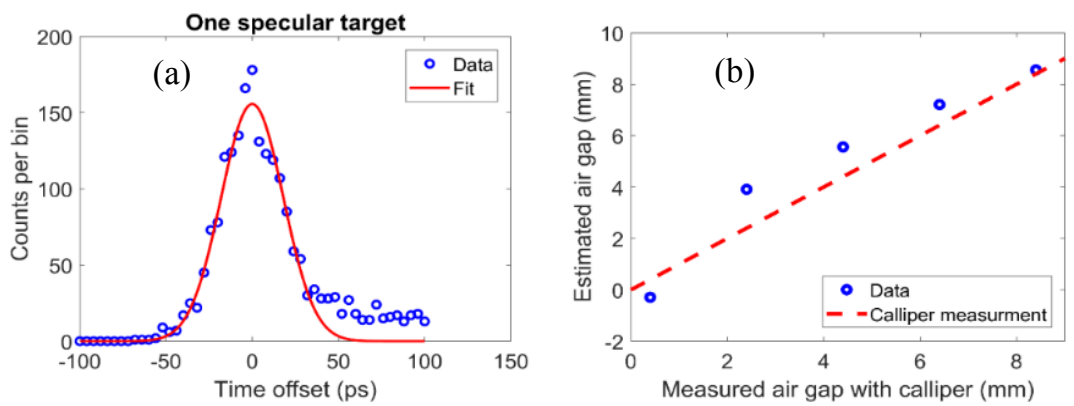

Fig. 1: (a) The measured signal from one specular target. The FWHM of the trace is 42 ps. The measured data is shown as circles and the solid line is a Gaussian fit. (b) Estimated air gap between the two targets.

To test the resolution of the system LIDAR traces were collected for multiple separations between two microscope slides varying from $0.4 \mathrm{~mm}$ to $8.4 \mathrm{~mm}$ of air and $1 \mathrm{~mm}$ of glass (thickness of the microscope slide).

The trace from a single reflection, as well as a Gaussian fit, can be seen in Fig 2 (a). The full width half maximum (FWHM) of a single reflection was only 42 ps. In Fig 2 (b) the estimated, through LIDAR, and the measured, by a caliper, air gap between the two microscope slides can be seen. An air gap separation of merely $0.4 \mathrm{~mm}$ (1.9 mm optical thickness) yielded a FWHM of $55 \mathrm{ps}$, which is $30 \%$ broader than for a single target.

\section{References}

1. R. W. Bukowski, "Smoke measurements in large- and small-scale fire testing - Part I," Fire Technol. 15(3), 173-179 (1979).

2. M. Henriksson, L. Allard, P- Jonsson, "Panoramic single-photon counting 3D lidar" Proc. SPIE 10796, 1079606 (2018).

3. J. Qiu, H. Xia, M. Shangguan, X. Dou, M. Li, Chong Wang, Xiang Shang, Shengfu Lin, and Jianjiang Liu, "Micro-pulse polarization lidar at $1.5 \mu \mathrm{m}$ using a single superconducting nanowire single-photon detector," Opt. Lett. 42, 4454-4457 (2017).

4. R. E. Warburton, A. McCarthy, A. M. Wallace, S. Hernandez-Marin, R. H. Hadfield, S. Woo Nam, and G. S. Buller, "Subcentimeter depth resolution using a single-photon counting time-of-flight laser ranging system at $1550 \mathrm{~nm}$ wavelength," Opt. Lett. 32, 2266-2268 (2007).

5. G. Temporão, S. Tanzilli, H. Zbinden, and N. Gisin, "Mid-infrared single-photon counting”' Opt. Lett. 31, 1094-1096 (2006).

6. J. Dam, P. Tidemand-Lichtenberg and C. Pedersen, "Room-temperature mid-infrared single-photon spectral imaging" Nat. Photonics. 6, 788, 2012.

7. H. Xia, G. S., M. Shangguan, X. Xia, X. Jia, C. Wang, J. Zhang, J. S. Pelc, M. M. Fejer, Q. Zhang, X. Dou, and J.-W. Pan, "Long-range micro-pulse aerosol lidar at $1.5 \mu \mathrm{m}$ with an upconversion single-photon detector," Opt. Lett. 40, 1579-1582 (2015).

8. $\quad$ L. Høgstedt, A. Fix, M. Wirth, C. Pedersen, and P. Tidemand-Lichtenberg, "Upconversion-based lidar measurements of atmospheric $\mathrm{CO}_{2}$," Opt. Express 24, 5152-5161 (2016). 